\title{
La investigación en la educación
}

\author{
Oscar Yecid Aparicio Gómez ${ }^{1}$ \\ Dra. Constanza Abadía García ${ }^{2}$
}

Recibido: 26-07-2018

Aceptado: 17-09-2018

\section{Resumen}

El presente artículo $^{3}$ pretende describir y analizar el proceso de introducción de la investigación como eje educativo de las TIC en una Institución Educativa del Municipio de Chía. La Institución Educativa, siguiendo la triple dimensión de su Proyecto Educativo Institucional, esto es: Investigación, Tecnología y Medio Ambiente, decidió designar el 20\% destinado a las áreas complementarias u optativas, para establecer la asignatura "Metodología de la Investigación" con una hora semanal de sesenta minutos en todos los grados escolares, desde Preescolar hasta Educación Media Académica (Prejardín a Décimo grado), Igualmente, decidió crear espacios académicos específicos para "Grupos de Investigación" con una hora semanal de sesenta minutos. Los Grupos de Investigación se ofrecen en las tres secciones escolares: Educación Preescolar, Educación Básica Primaria (de Primer grado a Quinto grado), Educación Básica Secundaria y Media Académica. Preescolar dispone de un Grupo de Investigación orientado por las tutoras y que versa sobre un tema de interés específico de su edad. Las secciones de Educación Básica Primaria y Educación Básica Secundaria y Media Académica, disponen

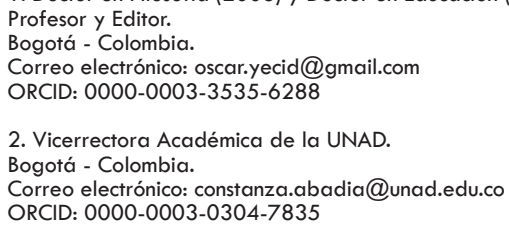

3. La información puede ampliarse en: Aparicio, Ó. Y. (2015). Las TIC como herramienta cognitiva para la investigación escolar (Tesis doctoral, Universitat de Barcelona, Barcelona, España). Recuperado de http://hdl.handle.net/10803/369830 
de cinco grupos cada una: Matemáticas, Ciencias Naturales, Ciencias Sociales, Lengua Castellana e Idioma Extranjero (inglés). Los estudiantes de cada sección pueden elegir el grupo al que desean pertenecer según estas cinco propuestas, y comparten el Proyecto de Investigación con los compañeros de su sección que comparten el mismo tema de interés.

Palabras clave:transferencia del conocimiento, TIC, instituciones educativas, colegio, investigación, metodología de la investigación, instrumentos de investigación, pedagogía.

\title{
Research in education
}

\begin{abstract}
This article aims to describe and analyze the process of introducing research as an educational axis of TIC in an Educational Institution of the Municipality of Chia. The Educational Institution, following the triple dimension of its Institutional Educational Project, this is: Research, Technology and Environment, decided to designate the 20\% destined to the complementary or optional areas, to establish the subject "Research Methodology" with one hour weekly sixty minutes in all grade levels, from Preschool to Academic Media Education (Pre-garden to tenth grade), also decided to create specific academic spaces for "Research Groups" with a weekly hour of sixty minutes. The Research Groups are offered in the three school sections: Preschool Education, Primary Basic Education (from First grade to Fifth grade), Basic Secondary Education and Academic Media. Preschool has a Research Group guided by the tutors and which deals with a topic of interest specific to their age. The sections of Primary Basic Education and Secondary and Academic Average Education, have five groups each: Mathematics, Natural Sciences, Social Sciences, Spanish Language and Foreign Language (English). The students of each section can choose the group they wish to belong to according to these five proposals, and they share the Research Project with the peers of their section who share the same topic of interest.
\end{abstract}

Keywords: knowledge transfer, TIC, educational institutions, school, research, research methodology, research instruments, pedagogy. 


\section{Introducción}

Esta introducción recoge el trabajo general que se ha realizado en el Centro Educativo con respecto a la asignatura "Metodología de la Investigación" en los diferentes espacios académicos. Posteriormente, se presentarán los resultados de la investigación aplicada en torno a los Proyectos de Investigación.

La investigación permite que los estudiantes obtengan información proveniente de una variedad de fuentes desde las cuales puedan construir su propia comprensión del mundo, orientados a través de un proceso de formación intelectual que les permite construir criterios y argumentos sobre su propio conocimiento y de esta manera lograr comprender crítica y racionalmente la realidad cotidiana que los rodea. Por lo tanto, se pretende que los estudiantes y en general la comunidad educativa, adopte una cultura investigadora con el apoyo y uso de las herramientas cognitivas que ofrecen y generan las TIC.

El objetivo general de la asignatura "Metodología de la Investigación" consiste en describir el proceso de generar una cultura de investigación en los estudiantes durante toda su formación escolar, motivando la formulación de preguntas desde el entorno próximo, y especialmente aquellas que corresponden a la etapa de desarrollo que atraviesan los estudiantes, según sus gustos e intereses, y mediante el uso de las TIC. La metodología de trabajo para el área de Investigación, para esta asignatura, se encuentra planteada en el Proyecto Educativo Institucional del Centro Educativo con el objetivo de privilegiar la construcción de conocimiento y la promoción de una actitud crítica frente al quehacer cotidiano, que comienza con la apropiación comprensiva de la realidad, y continúa con la transformación cualitativa de esta en los diversos ámbitos en los que se desenvuelve la vida de los estudiantes.

Para esta Investigación se propuso vincular el uso de las TIC como herramienta cognitiva en el desarrollo de la investigación realizada por los estudiantes, teniendo como base principal las preguntas de investigación que se derivan de los conocimientos adquiridos por los estudiantes en su vida diaria. Las preguntas planteadas por los profesores, fundamentadas, entre otros, en Pomar, Puig y Sbert (2000), y en Elder y Paul (2002), han sido abordadas desde información teórica y empírica analizada a partir de propuestas para interpretar la realidad en cada campo particular de 
los distintos saberes. El conocimiento generado a partir de este ejercicio no reproduce datos establecidos, sino que fragua nueva información desde la interacción entre el estudiante, el mundo y el conocimiento.

Los temas de investigación que se derivarán en proyectos formalmente constituidos, son propuestos por el profesor titular de cada asignatura en los distintos niveles académicos, y bajo la orientación metodológica del autor de esta tesis, así como de los demás expertos que han acompañado este proceso. Con el trabajo realizado por los estudiantes en la asignatura de "Metodología de la Investigación", se generan productos que recogen los avances en el proceso de investigación y pueden ser compartidos con sus pares, la comunidad educativa y sus familias. Los resultados obtenidos de estas investigaciones, son publicados en la Plataforma de Investigación del centro, con el objetivo de que pueda accederse a estos proyectos realizados a través de los años, permitiendo también que otros miembros de la comunidad educativa puedan consultar, profundizar y participar en los temas propuestos.

\section{Metodología}

El cuestionario está conformado por preguntas abiertas, y se compone de 16 preguntas dirigidas a los 10 profesores líderes de los Proyectos de Investigación. Cada una de las preguntas irá precedida por el número de Instrumento, el número de pregunta correspondiente, y además detallamos su objetivo.

02/P1. ¿Por qué considera que se debe realizar una investigación como la que propone? Objetivo: Analizar y exponer la razón por la que los Grupos de Investigación creen pertinente realizar la investigación elegida.

02/P2. ¿Cuál es el problema que se ha planteado en la investigación? Objetivo: Conocer el problema sobre el que se plantea la investigación que se va a trabajar.

02/P3. ¿Cuáles son los objetivos de la investigación? Objetivo: Distinguir los objetivos sobre los que se planteó la investigación que se va a trabajar. 
02/P4. ¿Qué preguntas ha planteado para la investigación? Objetivo: Identificar las preguntas que se han planteado inicialmente dentro del marco de esta investigación escolar.

02/P5. ¿Cuál es la hipótesis de la investigación? Objetivo: Conocer la hipótesis que se ha planteado para el Proyecto de Investigación.

02/P6. ¿Cuáles son los ejes fundamentales de la investigación? Objetivo: Reconocer los ejes fundamentales que se desarrollarán en el Proyecto de Investigación.

02/P7. ¿Por qué considera pertinente la formulación del Proyecto de Investigación? Objetivo: Reconocer la pertinencia del Proyecto de Investigación.

02/P8. ¿Por qué considera coherente la formulación del Proyecto de Investigación? Objetivo: Distinguir la coherencia planteada a la hora de proponer y desarrollar el Proyecto de Investigación.

02/P9. ¿Cuál es la aportación a la ciencia y la sociedad que se desea conseguir con el Proyecto de Investigación? Objetivo: Interpretar el Proyecto de Investigación a la luz del aporte que se puede brindar a la ciencia y a la sociedad.

02/P10. ¿Para qué usa las TIC en la investigación? Objetivo: Analizar el uso que se hará de las TIC para desarrollar la Investigación.

02/P11. ¿Qué herramientas TIC usará en la investigación? Objetivo: Conocer las herramientas TIC que planean usar los Grupos de Investigación en el marco de su proyecto.

02/P12. ¿Cuáles son las fases para el desarrollo de la investigación? Objetivo: Distinguir las fases sobre las que los Grupos de Investigación planean trabajar para la consecución de los objetivos esperados dentro del proyecto.

02/P13. ¿Cómo recogerán la información? Objetivo: Revisar la forma con la que los Grupos de Investigación planean recolectar la información de su Proyecto de Investigación. 
02/P14. ¿Cuáles son los tipos de información que se recogerán? Objetivo: Prever los tipos de información que se recogerán para la realización de este proyecto.

02/P15. ¿Qué técnicas o estrategias usarán para analizar e interpretar esta información? Objetivo: Considerar la manera como los grupos planean examinar la información recogida para incluirla desarrollar el Proyecto.

02/P16. ¿Cuál es el contexto sobre el que se incorporará esta investigación? Objetivo: Identificar el entorno y las condiciones donde se desarrollará el Proyecto de Investigación.

\section{Resultados}

La presentación de los resultados de este estudio se hará de manera descriptiva, mostrando las categorías que se obtuvieron en el análisis de tipo etnográfico y mencionando las respuestas más relevantes, y destacando entre comillas los términos y conceptos que corresponden a las categorías conceptuales más relevantes y significativas. Igualmente, se realiza un análisis sobre los resultados que se obtuvieron. Cuando se realice una citación textual sobre una respuesta de algún relator se enuncia con el prefijo $02 / R^{4}$ seguido por un número de acuerdo con la siguiente lista:

02/R1. Educación Preescolar:

Descubro lo maravilloso que es mi cuerpo

02/R2. Matemáticas. Educación Básica Primaria: El juego, un camino hacia las matemáticas

02/R3. Matemáticas. Educación Básica Secundaria: Las matemáticas y la vida: una relación imperceptible

02/R4. Lengua Castellana. Educación Básica Primaria: Creando conciencia, animales en vía de extinción

02/R5. Lengua Castellana. Educación Básica Secundaria: Literatura en vía de extinción

4. El 02 hace referencia al instrumento que se ha aplicado y "R" corresponde al número asignado al relator. 
02/R6. Ciencias Naturales. Educación Básica Primaria: Cultivos hidropónicos y agricultura sostenible

02/R7. Ciencias Naturales. Educación Básica Secundaria: Lombricultivo y su efecto en los alimentos orgánicos

02/R8. Inglés. Educación Básica Primaria, Educación Básica Secundaria y Educación Media Académica: Welcome to Colombia

02/R9. Ciencias Sociales. Educación Básica Primaria: La deforestación en Colombia

02/R10. Ciencias Sociales. Educación Básica Primaria: Visto y no visto

Las preguntas del cuestionario se enuncian con el prefijo 02/ $\mathrm{P}^{5}$ seguido por un número:

\section{2/P1. ¿Por qué considera que se debe realizar una investigación como la que propone?}

En las respuestas a esta pregunta se pudo observar que todos los profesores hablaban, bien sea de forma explícita o implícita, de "necesidades". Estas necesidades de las que hablan los profesores en sus respuestas se pueden agrupar en: "necesidades en el mundo", "necesidades culturales", "necesidades propias del contexto de los estudiantes" (Langer, 2016) o "necesidades de los propios estudiantes" (Tahull, 2016). A pesar de estas necesidades variadas, las justificaciones de las investigaciones parecen mostrar que los profesores opinan que debe propenderse una necesidad de cambio, bien sea en la idiosincrasia propia del estudiante o afectando también la de su entorno cercano.

En estas respuestas se pueden resaltar las respuestas que dieron los profesores titulares de matemáticas (02/R2: El juego, un camino hacia las matemáticas y 02/R3: Las matemáticas y la vida: una relación imperceptible) ya que justifican la investigación que van a realizar en la necesidad de mejorar la relación estudiante-matemáticas. Uno se basa el contexto del estudiante: "La anticultura matemática en la sociedad y el gran pánico

5. El 02 hace referencia a las preguntas correspondientes al instrumento que se ha aplicado $y$ " $R$ " corresponde al número y orden de la pregunta que se ha realizado. 
cultural que existe por el tema de las matemáticas" (02/R3: Las matemáticas y la vida: una relación imperceptible), mientras que el otro se basa directamente en los estudiantes: "Las matemáticas suelen convertirse en el dolor de cabeza de muchos estudiantes" (02/R2: El juego, un camino hacia las matemáticas). Esto puede significar que la diferencia entre los dos es que explican el porqué de su investigación por la experiencia social ${ }^{6}$ que han tenido con el problema (02/R3: Las matemáticas y la vida: una relación imperceptible) y con la experiencia directa que ha tenido con los estudiantes (02/R2: El juego, un camino hacia las matemáticas).

Las investigaciones que proponen los profesores parten de las necesidades que perciben en su entorno, o en el entorno de los estudiantes. Esto es muy interesante ya que significa que quieren centrarse en lo que está cerca de los estudiantes, en el contexto próximo, vivencial y experiencial, no quieren resolver problemas fuera del alcance de los estudiantes, lo que en últimas genera que los estudiantes se sientan cómodos con la investigación que van a emprender.

\section{2/P2. ¿Cuál es el problema que se ha planteado en la investigación?}

Los profesores manifestaron que van a trabajar sobre las "problemáticas de actualidad". En las respuestas de los profesores, sobresalen las que hablan de "falta de conciencia sobre la afectación" y el "desconocimiento"7 de la humanidad en diversos campos, esto debido a que en los problemas se encuentran algunos referentes a la deforestación, el manejo de material orgánico, la extinción de los animales y la literatura "basura" gracias a las necesidades del mercado (de Almeida, Santos, Porto, 2017).

Uno de los problemas más llamativos, es sobre el que va a trabajar el Grupo de Investigación de Ciencias Sociales de Educación Básica Secundaria (02/R10: Visto y no visto), ya que el proyecto se basa "en los casos en los que las imágenes se analizan en el texto, su testimonio suele utilizarse para ilustrar las conclusiones a las que el autor ha llegado por otros medios, y no para dar nuevas respuestas o plantear nuevas cuestiones", este es un problema sobre la pérdida de la información que puede encontrarse en las imágenes por no tomarlas muy en cuenta como instrumento para la investigación (Konieczny, 2015).

6. Social refiriéndome a familiares, amigos, estudiantes y lo que escucha de la sociedad.

7. Algunos lo hacen de forma explícita, mientras en las respuestas de otros se puede notar que hacen referencia a estos términos. 
De los problemas que van a trabajar los diferentes Proyectos de Investigación pudo verse que, en su mayoría, son problemas cercanos a los estudiantes ya que puede que hayan oído hablar de ellos en los diversos medios de comunicación ${ }^{8}$ o que son problemas que están presentes en sus vidas (Pérez, 2014). En este último tipo de problema están los que hablan sobre el desconocimiento del cuerpo por parte de los estudiantes más pequeños y los problemas de aprendizaje que se tiene con las matemáticas. De acuerdo con esto, es posible afirmar que los profesores quieren basar sus investigaciones en problemas cercanos a los estudiantes (Pérez, 2013), de los cuales tengan ciertas nociones y tal vez algún tipo de interés, y que estén contextualizados y sean significativos.

\section{2/P3. ¿Cuáles son los objetivos de la investigación?}

Evidentemente, los objetivos de cada línea de investigación se basan en un marco conceptual propio de cada una de las materias que representan, pero en las respuestas es claro ver que el generar conocimiento en los estudiantes es el factor común en las respuestas. Indiscutiblemente cada uno lo dice a su manera y refiriéndose directamente al tema de su línea de investigación, pero es posible inferirlo de las respuestas.

Otro de los objetivos comunes, aunque no se encuentran en todas las respuestas, es "generar o afianzar habilidades en los estudiantes". En las respuestas que hablan sobre este tema, los profesores manifestaron que querían desarrollar "habilidades analíticas", en los estudiantes, propias del tema de su investigación o habilidades que son necesarias para el desarrollo del Proyecto de Investigación.

"Concebir productos o soluciones" también es uno de los objetivos comunes en la mayoría de los Proyectos de Investigación, en este objetivo se pueden agrupar las respuestas referentes a la búsqueda de soluciones a la problemática que se planteó ${ }^{9}$ y aquellas respuestas en las que los profesores manifestaron que un objetivo era que los estudiantes "crearan un producto tangible" que contribuyera a resolver la problemática planteada.

Algunos de los profesores manifestaron en sus respuestas que su objetivo con el Proyecto de Investigación era "generar conciencia" o "lograr

8. Medios de comunicación en referencia a todo tipo de fuente de información a la que están expuestos los estudiantes para no excluir a los medios de comunicación no tradicionales como las redes sociales.

9. En la mayoría de las respuestas no lo hacen de manera explícita pero es fácil inferirlo. 
un cambio en la manera de pensar", comenzando por los estudiantes y la institución para llegar a un nivel mayor (algunos hacían referencia a la sociedad), sobre la problemática (Ferreyra, 2014) y las posibles soluciones existentes.

Entre las respuestas más llamativas sobre esta pregunta, se encuentran dos que se refieren directamente a la concepción de un producto y que necesitan que los estudiantes desarrollen habilidades que normalmente no poseen los estudiantes que lo realizarán, estas son: "Desarrollar un juego donde se apliquen conceptos matemáticos" (02/R2: El juego, un camino hacia las matemáticas) y "Aprovechar los residuos orgánicos mediante la técnica de lombricultura para la producción de abono en el Colegio" (02/R7: Lombricultivo y su efecto en los alimentos orgánicos). En la primera respuesta (02/R2: El juego, un camino hacia las matemáticas), el profesor manifestó que quiere que los estudiantes realicen un juego virtual matemático; en la segunda (02/R7: Lombricultivo y su efecto en los alimentos orgánicos), la profesora manifestó que quiere, que junto con los estudiantes, se haga un lombricultivo.

Lo más significativo en las respuestas a esta pregunta es que, en su mayoría, los profesores opinan que el primer objetivo es que los estudiantes conozcan sobre lo que están trabajando, para lograr "generar conciencia" o generar un cambio en la idiosincrasia. Otro aspecto a resaltar es que todos los profesores en sus objetivos tienen la elaboración de un producto de la investigación, puede ser intangible como la concepción de una solución o tangible como un juego.

\section{2/P4. ¿Qué preguntas ha planteado para la investigación?}

En las preguntas planteadas por parte de los profesores, se puede ver que todos proponen al menos, una "pregunta conceptual" sobre el tema de investigación. Además, la mayoría propone una pregunta haciendo referencia a cómo solucionar la problemática, a cómo lograr concientizar a las personas o cómo se puede aportar para solucionar la problemática.

En las preguntas conceptuales se pueden resaltar las que hacen referencia a soluciones ya existentes y cómo aplicarlas en el contexto que busca el Proyecto de Investigación, por ejemplo: "¿Qué beneficios adicionales ofrecen los cultivos hidropónicos en los hogares colombianos?" (02/R7: Lombricultivo y su efecto en los alimentos orgánicos). Además, también hay preguntas que plantean los profesores que hacen referencia 
a bases teóricas que necesitan los estudiantes y que son necesarias durante el desarrollo del Proyecto de Investigación.

De las preguntas planteadas por los profesores, se puede ver que lo que quieren primero es que se sienten bases teóricas sobre el tema del Proyecto de Investigación, luego difieren un poco, pero se ve que hay algunos que quieren que los estudiantes busquen una solución para la problemática, otros quieren que hagan un aporte para mejorar la problemática (una solución parcial) y otros quieren que elaboren productos para concientizar sobre la problemática y la posible solución.

\section{2/P5. ¿Cuál es la hipótesis de la Investigación?}

En las hipótesis de los Proyectos de Investigación, la "variable" sobre la que los profesores quieren realizar cambios para el estudio es el "conocimiento de la población de estudio". Esto ocurre en la mayoría de los casos, pero hay algunos que incluyen otras variables sobre las que quieren realizar cambios como la "Motivación" en la población de estudio. Esta variable se puede ejemplificar en las hipótesis como "Los estudiantes que logran conocer las matemáticas como un elemento fundamental en su entorno" (02/R4: Creando conciencia, animales en vía de extinción), "Construir unos criterios propios y personales sobre la literatura" (02/ R5: Literatura en vía de extinción) o "Por medio del conocimiento sobre la agricultura sostenible" (02/R6: Cultivos hidropónicos y agricultura sostenible).

En otras variables hay una mayor diversidad de respuestas, pero todas tienden a apuntar sobre la mentalidad de las personas o a generar cambios en cómo las personas realizan algunas cosas. De hecho, algunas de las hipótesis están asociadas a que se genera un cambio en la mentalidad de los estudiantes, a que se generan cambios en la mentalidad de la sociedad, a generar conciencia sobre ciertos problemas o simplemente a generar ciertos comportamientos en la muestra participante. Esto se evidencia en las hipótesis: "se puede generar conciencia sobre este grave problema" (02/R4: Creando conciencia, animales en vía de extinción), "el proyecto influye de forma directa e interpretación de conceptos, términos e ideas de diferentes contextos y hechos, en un proceso de transformación económica, social o política" (02/R10: Visto y no visto), "lograr que un grupo de viajeros recién Ilegados a Colombia se adapten rápidamente a la cultura propia" (02/R8: Welcome to Colombia) o "se 
genere conciencia acerca del cuidado que deben tener hacia el entorno" (02/R10: Visto y no visto).

\section{2/P6. ¿Cuáles son los ejes fundamentales de la investigación?}

En cuanto a los ejes que los profesores consideran fundamentales en sus Proyectos de Investigación, se puede notar que sus planteamientos se pueden agrupar en tres categorías. La primera, se refiere a "los ejes temáticos de la investigación" en sí, esto alude a que los profesores plantean ejes en los que se buscaba una fundamentación teórica sobre la investigación como "historia de las matemáticas" (02/R3: Las matemáticas y la vida: una relación imperceptible) o "historia, géneros, influencia de la cultura y sociedad" (02/R5 Literatura en vía de extinción). La segunda, se refiere a "la fundamentación sobre el producto" que van a crear, en esta categoría se encuentran los ejes referentes al porqué realizar un producto como el que proponen los profesores, cómo realizar el producto, o qué productos se pueden crear en el Proyecto de Investigación entre estos están: "Cómo llevar el mensaje" (02/R4: Creando conciencia, animales en vía de extinción) y "Actividades a realizar en la granja" (02/R7: Lombricultivo y su efecto en los alimentos orgánicos); de esta categoría se puede resaltar que no todos los Proyectos de Investigación lo tienen en su planteamiento. La tercera, se refiere a "la concepción de los productos" como "desarrollo de juego lúdico" (02/R2: El juego, un camino hacia las matemáticas) o "trabajo práctico en la huerta hidropónica" (02/R6: Cultivos hidropónicos y agricultura sostenible).

Con respecto a los "ejes temáticos", es destacable el hecho de que existan categorías tan marcadas en las propuestas de los Proyectos de Investigación, esto dado que cada profesor trabaja una línea de investigación diferente y cada una de estas está enmarcada en un área del conocimiento diferente. A pesar de esto, es congruente dado que están relacionadas con las respuestas que han dado en las preguntas anteriores, se mantiene la tendencia que se ha podido ver de tener una relación conocimientoproducto.

\section{2/P7. ¿Por qué considera pertinente la formulación del Proyecto de Investigación?}

En las respuestas dadas en esta pregunta por parte de los profesores, como en 02/R1: Descubro lo maravilloso que es mi cuerpo, se puede resaltar que todos hablan de necesidades, estas son de nivel personal (estudiantes), contextual, cultural o global. Pero todas las necesidades de 
las que hablan son muy cercanas a los estudiantes, bien sea porque están presentes en sus vidas o porque se supone que conocen de ellas.

Estas necesidades, normalmente, hablan de un cambio en la forma de pensar o en la forma en la que se hacen las cosas, pero lo más destacable sobre esto, es cómo los profesores hablan de la necesidad de generar estos cambios en los estudiantes dada su edad y su capacidad de generar cambios en otras personas en un futuro. Esto, según lo que indican los profesores, se debe a que, tal como se afirma en "Los niños de ahora serán los ciudadanos del futuro" (02/R3: Las matemáticas y la vida: una relación imperceptible $)^{10}$.

Esto muestra que, para los profesores, la pertinencia de sus proyectos se basa en la formación de los ciudadanos del futuro en las problemáticas propias de sus Proyectos de Investigación. Esto teniendo en mente la concientización sobre la base de la sociedad futura, los niños.

\section{2/P8. ¿Por qué considera coherente la formulación del Proyecto de Investigación?}

Los profesores en sus respuestas daban a entender que la coherencia se basaba en la relación entre la justificación, la problemática, los objetivos, las preguntas, la hipótesis y los ejes fundamentales dentro sus Proyectos de Investigación. Además, algunos también hablaron del uso de las TIC que darán en los proyectos y de las fases del desarrollo de sus Proyectos de Investigación.

De las respuestas de los profesores sobre la coherencia del planteamiento de su Proyecto de Investigación, es claro ver como ellos expresan que cada uno de los ítems en el planteamiento de cada Proyecto de Investigación está basado en otro ítem. Por ejemplo, "el hecho de que busquemos con este Proyecto de Investigación fomentar el estudio de las matemáticas está basado en el problema en sí, el que tienen los estudiantes que es su temor a las matemáticas, se puede decir que el objetivo es la finalidad de la solución de la problemática" (02/R2: El juego, un camino hacia las matemáticas).

Con respecto a esto, es destacable que en las respuestas plasmadas en este documento y especialmente las categorías resultantes del

10. Aunque la citación en el texto corresponde a 02/R3: Las matemáticas y la vida: una relación imperceptible, todos los profesores hacen alusión, de manera explícita o implícita al mismo tema. 
análisis, efectivamente, se encuentra una coherencia. Esto debido a que, desde la explicación de los problemas, los objetivos, las preguntas de la investigación, la hipótesis y los ejes fundamentales del Proyecto de Investigación, se viene manteniendo una relación de necesidad (relativa a la problemática) -conocimiento-productos-generación de conciencia.

\section{2/P9. ¿Cuál es la aportación a la ciencia y la sociedad que se desea conseguir con el Proyecto de Investigación?}

La mayoría de los profesores respondieron respecto a la aportación a la sociedad antes que a la ciencia del Proyecto de Investigación (Aparicio, 2018), de hecho, el único proyecto donde se habla de una aportación a la ciencia es en el Proyecto de Investigación de ciencias sociales de Educación Básica Secundaria, ya que según 02/R10: Visto y no visto, el proyecto "aporta profundizando en esta temática que plantea una nueva forma de realizar investigaciones sociales".

Con respecto a la aportación a la sociedad (de la calle, Malaver, Gallego, Rodríguez, Flórez, Saldaña, 2014), los profesores hablan sobre un "aporte positivo", ya que buscan formar estudiantes conscientes de las problemáticas propias de los Proyectos de Investigación y con nociones de cómo "concientizar" a otros (aquellos Proyectos de Investigación que hacen referencia a buscar entregar un mensaje de concientización sobre la problemática y las posibles soluciones). Esto es claro al ver algunas respuestas como "concientizar por medio de los productos de la investigación sobre la reforestación y sobre el cuidado del medioambiente" (02) R9: La deforestación en Colombia) o "generará conciencia en cualquier persona y una posición crítica frente a la situación actual del mundo" (02/ R4: Creando conciencia, animales en vía de extinción).

Las respuestas de los profesores a esta pregunta no salen de la tendencia que se lleva, ya que se mantienen en lo que respecta a generar conciencia en los estudiantes y que estos puedan concientizar a su entorno sobre las problemáticas y posibles soluciones propias de los Proyectos de Investigación.

\section{2/P10. ¿Para qué usará las TIC en la investigación?}

El principal uso que los profesores le darán a las TIC es el de "fuente de información", pero de dos formas diferentes. Primero, algunos la usarán para "consultar información" existente en Internet bien sea como forma 
de fundamentación teórica, para informarse de lo que se está haciendo en el campo en otras investigaciones o simplemente como forma de "búsqueda de información pertinente" a la investigación. Por otro lado, algunos manifestaron que las usarán como forma de "captar información", esto significa que usarán encuestas distribuidas por Internet o por medio de "encuestas por videocámara" (02/R8: Welcome to Colombia).

De forma complementaria al uso anterior, los profesores también expresaron que usarán las TIC para organizar y analizar la información consultada, aunque no era una respuesta homogénea en las respuestas de los profesores. Otra de las respuestas provistas por los profesores, fue hacer uso de las TIC como una forma de "seguimiento del desarrollo del proyecto" por parte de los estudiantes, aunque es una respuesta minoritaria.

Finalmente, los profesores hicieron referencia en sus respuestas a que usarán las TIC para la "elaboración de los productos", tanto finales como parciales, de la investigación y para su posterior "divulgación"11. En este grupo de respuestas, se encuentran algunas referentes al propio proyecto como la "realización de los videos de los sitios históricos colombianos, su edición y luego compartirlo en YouTube. Luego, se usarán los enlaces para crear los códigos QR y poder colocarlos en el mapa de Colombia" (02/R8: Welcome to Colombia) ${ }^{12}$. Posteriormente, los profesores buscarían compartir los resultados (productos) de los Proyectos de Investigación para que puedan ser consultados por cualquier Grupo de Investigación, tanto interno como externo.

Estos usos de las TIC expuestos por los profesores coinciden con las fases básicas de las Competencias Informacionales (Lau, 2007):

- Acceso y valoración de información: "fuente de información", "consultar información", "búsqueda de información pertinente

- Procesamiento de información: "seguimiento del desarrollo del proyecto", "elaboración de los productos"

- Uso y comunicación de información: "divulgación"

Así mismo, el uso que le darán a las TIC en los Proyectos de Investigación se puede ver en una relación información-análisis-

11. Los profesores expresaban que usarían las TIC para presentar los resultados de los Proyectos de Investigación.

12. Cada proyecto tiene en su concepción productos diferentes pero el ejemplo es ilustrativo. 
productos-distribución. Esto es congruente con lo que se ha planteado en las preguntas anteriores solo que en estas respuestas pareciera que se profundizara en cómo es la generación de conocimiento (informaciónanálisis) y a la manera de generar conciencia (distribución).

\section{2/P11. ¿Qué herramientas TIC usará en la investigación?}

La principal herramienta que se usará en los Proyectos de Investigación es Internet ${ }^{13}$ para la Consulta de Información. Por otro lado, para la captación de información algunos de los profesores manifestaron que usarán las encuestas de Google Drive y Skype.

Otra de las herramientas que manifestaron que usarán en los Proyectos de Investigación fueron las wikis, ya que estas tienen usos variados, desde la organización de la información consultada, pasando por el seguimiento de los proyectos, hasta la distribución de los resultados del Proyecto de Investigación en la comunidad. Además, también manifestaron que usarán los blogs y los foros para la distribución de resultados.

Para el análisis de la información, los profesores plantearon en sus Proyectos de Investigación el uso de generadores de mapas conceptuales, herramientas de análisis estadístico y para graficar los resultados ( $E x$ cel).

Sobre la elaboración de los productos, los planteamientos de los profesores son divididos, esto dada la naturaleza de los productos que esperan obtener. La mayoría expresaron que usarán los procesadores de texto (Word) y los programas de presentación de diapositivas (PowerPoint o Prezi), mientras que los que dependen de la naturaleza del producto agrupan: aplicaciones para desarrollar juegos, programas de edición de videos, YouTube (para compartir los videos), programas de edición de imágenes, programas de codificación de códigos $Q R$ y programas de creación de cómics.

\section{2/P12. ¿Cuáles son las fases para el desarrollo de la investigación?}

A pesar de que las fases que plantean los profesores en los proyectos difieren un poco, especialmente por los nombres de las fases y qué realizarán en cada fase, es posible ver una inclinación a una misma línea. Cabe

13. No se tienen en cuenta las aplicaciones que existen en Internet, simplemente el acceso a Internet para consultar información, por tanto, podemos suponer que mayoritariamente se refieren a buscadores. 
aclarar que hay algunas fases que se enunciarán a continuación que no aparecen en todos los planteamientos de los proyectos, pero sí lo hacen en la mayoría.

Fase de acercamiento a los estudiantes, en esta fase los profesores les comentan a los estudiantes sobre el proyecto, la problemática, los objetivos, las preguntas, la hipótesis, lo que van a trabajar y buscan que los estudiantes conozcan sobre el proyecto. Algunos profesores también manifestaron que en esta fase recibirán comentarios de los estudiantes para identificar conocimientos previos y buscar mejorar el planteamiento del proyecto.

Fase metodológica, en esta fase los profesores les explican a los estudiantes las tareas que deben realizar en las diversas fases del proyecto, se realiza la división de tareas y se define la manera en la que van a trabajar (definido por el profesor, usualmente). En caso de que tengan que elegir alguna temática o algún elemento en la investigación se encontraría en esta fase también.

Fase de fundamentación teórica, en esta fase los estudiantes se impregnan de todos los conocimientos necesarios para continuar con el proyecto.

Fase de recolección de datos, en esta fase los estudiantes en compañía de los profesores recopilan los datos necesarios para continuar con las siguientes fases ${ }^{14}$ esta fase en algunos de los proyectos ocurrirá en paralelo con las siguientes fases, para que el proyecto se mantenga alimentado de información.

Fase de análisis, en esta fase los estudiantes en compañía del profesor realizan el análisis de los datos y de la información recopilada.

Fase de preproducción, esta fase no es homogénea en los proyectos, pero es necesaria en algunos, dada la necesidad de consultar sobre herramientas que usarán en la fase de producción o la necesidad de definir los productos que realizarán.

Fase de producción, en esta fase los estudiantes en compañía de los profesores realizarán los productos de los proyectos de acuerdo con los productos definidos. Algunos proyectos en esta misma fase tienen una

14. Esta recopilación puede ser consulta de información o captación de información como la realización de encuestas. 
fase de experimentación con los productos que se originaron y se volvería a la fase de análisis de datos.

Fase de divulgación de los resultados, en esta fase se le presentarán a la comunidad los resultados, algunos definieron la realización de campañas de concientización, otros simplemente harán públicos sus resultados por medio de las wikis y los blogs, mientras que en otros proyectos se planteó la exposición de los resultados a los estudiantes y profesores del Colegio.

\section{2/P13. ¿Cómo recogerán la información?}

La mayoría de los profesores comentaron que la información en su Proyecto de Investigación se recolectará por la Consulta de Información por parte de los estudiantes, algunos dijeron que la información consultada por los estudiantes debían buscarla en Internet. Otras formas de recolección de información que aparecen en las respuestas de los profesores son: el registro de observaciones ${ }^{15}$ y la realización de encuestas y/o entrevistas.

\section{2/P14. ¿Cuáles son los tipos de información que se recogerán?}

Algunos de los profesores se expresaron de manera muy general y respondían que recogerían datos cuantitativos y/o cualitativos. Por otro lado, hubo otros cuyas respuestas eran más profundas y respondían que la información que se recogería en los proyectos era consultada por los estudiantes, información de opinión y percepción de diferentes personas, información de la observación, información de la experiencia de los propios estudiantes e información de experimentación.

\section{2/P15. ¿Qué técnicas o estrategias usarán para analizar e interpre- tar esta información?}

Algunos de los profesores sobre los que se realizó el estudio respondieron que su planteamiento con respecto a este aspecto es un "acompañamiento al estudiante para el análisis de la información", de esta forma, según los profesores, se realiza un análisis por parte del estudiante con un experto en el tema que lo guíe para poder lograr mejores resultados y evitar que el estudiante se pierda en el análisis que está realizando. Por otro lado, algunos de los profesores manifestaron que el análisis debía ser "dependiente de las opiniones, conocimientos y suposiciones de los

15. En este método de recolección se tiene en cuenta el registro de la experiencia propia al entrar en contacto con cierto fenómeno. 
estudiantes" (02/R6: Cultivos hidropónicos y agricultura sostenible), o sea, mediatizado por sus conocimientos previos, para que ellos realicen por su propia cuenta el análisis de la información recopilada.

Otras de las respuestas que daban los profesores sobre este aspecto, hablan sobre los análisis por medio de métodos estadísticos, por medio de un análisis cualitativo y socialización de los resultados.

La respuesta más llamativa es una que se refiere a la socialización de la información por medio de "mesas redondas, exposiciones, charlas y discusiones para confrontar lo consultado" (02/R1: Descubro lo maravilloso que es mi cuerpo), de este modo se busca que los estudiantes analicen la información en conjunto para lograr tener un panorama más amplio y lograr mejores conclusiones.

\section{2/P16. ¿Cuál es el contexto sobre el que se incorporará esta inves- tigación?}

El contexto de las investigaciones es bastante variado, hay algunas de las investigaciones que apuntan a la población mundial, mientras hay otras que se contextualizan en la población colombiana, la población estudiantil colombiana, la población urbana colombiana o los estudiantes de Preescolar colombianos.

En cuanto a las muestras sobre las que se trabajará en los diversos Proyectos de Investigación, estas se encuentran enmarcadas en los estudiantes de Preescolar del Colegio Padre Manyanet - Chía, los estudiantes de Educación Básica Primaria del Colegio Padre Manyanet - Chía, los estudiantes de Educación Básica Secundaria del Colegio Padre Manyanet - Chía o los estudiantes del Colegio Padre Manyanet - Chía en general. Cabe destacar que en algunos de los Proyectos de Investigación también se incluyen en la muestra las familias de los estudiantes.

\section{Conclusiones}

Los Proyectos de Investigación propuestos por los profesores del Colegio Padre Manyanet - Chía buscan estar íntimamente relacionados con los estudiantes que pertenecen al Grupo de Investigación, al menos esto puede percibirse en el planteamiento que proponen. Por un lado, las 
problemáticas y las necesidades en las que están basados los Proyectos de Investigación se han planteado cercanos a los estudiantes, bien sea porque las problemáticas y necesidades son propias, propias de su contexto o de las cuales los estudiantes están informados (problemáticas mundiales, especialmente). Por otro lado, en las fases de desarrollo de la investigación los profesores manifestaban el planteamiento de una fase de acercamiento a los estudiantes, en la cual se buscaba que los estudiantes conocieran del proyecto y de sus planteamientos, además, algunos de los profesores proponían que los estudiantes eligieran ciertas temáticas o elementos en la investigación ${ }^{16}$. Esto teniendo que cuenta que los profesores buscan que los estudiantes tengan ciertas nociones y también posean cierto interés por la temática de la investigación.

En el planteamiento de los Proyectos de Investigación, los profesores expresaron que buscan primero que los estudiantes sienten bases teóricas sobre las temáticas del Proyecto de Investigación, luego buscan que basados en esta teoría los estudiantes elaboren diversos productos ${ }^{17}$ y que estos productos, en la mayoría de los Proyectos de Investigación, sirvan para concientizar sobre la problemática y sobre cómo aportar para solucionarla. Estas intenciones se pueden ver a lo largo de las respuestas de los profesores sobre el planteamiento de sus Proyectos de Investigación, de hecho, se puede ver una relación Problemática (necesidades) - Conocimiento (Consulta de Información-análisis de información) - Productos - Concientización (divulgación y compartir resultados), en las respuestas de los profesores a algunas preguntas se veía una dedicación o una profundización a ciertos ítems de esta lista. En general, la mayoría de los proyectos siguen la misma línea y difieren simplemente en detalles.

Uno de los aspectos más destacables en el planteamiento de los diferentes Proyectos de Investigación es que la mayoría de los profesores opinan que sus Proyectos de Investigación aportarán a la sociedad y son pertinentes porque tienen en mente la concientización de la base de la sociedad futura (especialmente por la información que les brinda el Proyecto de Investigación), los niños. Esto basándose en que haciendo un cambio en la mentalidad de los niños se realiza un cambio social en el futuro.

16. Estos son, por ejemplo, elegir el animal a estudiar, elegir el texto a estudiar, etc.

17. En los productos se encuentran las posibles soluciones o una aportación para la solución de la problemáTICa, como productos intangibles. Mientras tanto, otros proponen la realización de productos tangibles como juegos, una huerta hidropónica en el Colegio, entre otros. 
En cuanto a la información, su tratamiento y análisis, los profesores manifestaron que en sus planteamientos se encontraba la información consultada por estudiantes, la información referente a la opinión y percepción de ciertas personas, la información de las observaciones de los estudiantes y la información de la experimentación. La información la recogerán por búsqueda de información de Internet, entrevistas, encuestas y observación. Mientras que, para analizarla, en los planteamientos de los Proyectos de Investigación, los profesores manifestaron que el análisis será realizado por los estudiantes y algunos opinan que debe ser guiado, mientras que otros opinan que no debe ser guiado, las técnicas que usarán serán los métodos estadísticos, análisis cualitativo y socialización de información.

Con respecto al contexto de los Proyectos de Investigación, estos están planteados para estar dirigidos desde la población mundial hasta los estudiantes de Preescolar colombianos, pasando por la población colombiana, la población escolar colombiana y la población urbana colombiana. Mientras que la muestra de los proyectos son los estudiantes del Colegio, algunos se circunscriben a los estudiantes de Educación Básica Primaria, a los estudiantes de Educación Básica Secundaria o a los estudiantes de Preescolar.

Uno de los aspectos más interesantes en los Proyectos de Investigación es la interdisciplinariedad de algunos de ellos. Mientras hay unos que lo hacen de manera explícita, como las investigaciones que pertenecen a cierta área del conocimiento, pero serán trabajados por otras, hay otras que necesitan de otras áreas. Esto muestra cómo en algunos de los proyectos, es necesario incluir otras áreas del conocimiento para poder profundizar en lo que los profesores desean trabajar en los Proyectos de Investigación. 


\section{Referencias bibliográficas}

Aparicio, Ó. Y. (2015). Las TIC como herramienta cognitiva para la investigación escolar (Tesis doctoral, Universitat de Barcelona, Barcelona, España). Recuperado de http://hdl. handle.net/10803/369830

Aparicio, O.Y. (2018). La investigación escolar. Revista Interamericana de Investigación, Educación y Pedagogía, RIIEP, 11(2). https://orcid.org/0000-0003-3535-6288

de Almeida, R. R., Santos, M. F., \& Porto, J. C. (2017). Lectura de textos ficcionales y el enfoque escolar de literatura: contribuciones para una Pedagogía de la Elección. Revista Interamericana de Educación, Pedagogía y Estudios Culturales, 9(1), 35-51. DOI: https:// doi.org/10.22490/25391887.1925

de la Calle, C. V., Malaver, M. O., Gallego, J. D. M., Rodríguez, M., Flórez, J. C., Henao, C. E. \& Saldaña, R. (2014). Aportes de los doctorados de educación en ciencia, tecnología y sociedad, desde la sistematización de sus investigaciones doctorales científicas y formativas, 2000-2010. Revista Interamericana de Investigación, Educación y Pedagogía, RIIEP, 7(1). DOI: https://doi.org/10.15332/s1657-107X.2014.0001.04

Elder, L. y Paul, R. (2002). El Arte de Formular Preguntas Esenciales. Sonoma: Sonoma State University, Foundation for Critical Thinking. Recuperado de: https://www. criticalthinking.org/resources/PDF/SP-AskingQuestions.pdf

Ferreyra, H. A. (2014). Mesas Socioeducativas para la Inclusión y la Igualdad. Un programa "De todos con todos". Una experiencia en construcción. Revista Interamericana de Investigación, Educación y Pedagogía, RIIEP, 7(2). DOI: https://doi.org/10.15332/s1657107X.2014.0002.01

Konieczny, P. (2015). Lorenzo García Aretio: bases, mediaciones y futuro de la educación a distancia en la sociedad digital. Revista Interamericana de Investigación, Educación y Pedagogía, RIIEP, 8(1). DOI: https://doi.org/10.15332/s1657-107X.2015.0001.08

Langer, E. (2016). La construcción de confianza para el estudio de prácticas de resistencia en la escolarización de jóvenes en contextos de pobreza urbana. Revista Interamericana de Investigación, Educación y Pedagogía, RIIEP, 9(2). DOI: https://doi. org/10.22490/25391887.1945

Lau, Jesús. (2007). Directrices sobre desarrollo de habilidades informativas para el aprendizaje permanente. Boca del Río, Veracruz, México: IFLA. Recuperado de: http:// www.ifla.org/files/assets/information-literacy/publications/ifla-guidelines-es.pdf

Pérez, T. H. P. (2013). Aproximaciones al estado de la cuestión de la investigación en educación y derechos humanos. Revista Interamericana de Investigación, Educación y Pedagogía, RIIEP, 6(1). DOI: https://doi.org/10.15332/s1657-107X.2013.0001.05

Pérez, T. H. (2014). Colombia: de la educación en emergencia hacia una educación para 
el posconflicto y la paz. Revista Interamericana de Investigación, Educación y Pedagogía, RIIEP, 7(2). DOI: https://doi.org/10.15332/s1657-107X.2014.0002.06

Pomar, M., Puig, I. de, y Sbert, M. (2000). Per què és interessant fer preguntes? Butlletí Filosofia 3/18, (39-40), 4-7. Recuperado de:

http://www.grupiref.org/cat/articles-filosofia/per-que-es-interessant-fer-preguntes.pdf

Tahull, J. (2016). Modernidad, educación y género. El proyecto inacabado. Revista Interamericana de Investigación, Educación y Pedagogía, RIIEP, 9(2), 159-178. DOI: https://doi.org/10.22490/25391887.1947 